\title{
A HILBERT CUBE L-S CATEGORY
}

\author{
RAYMOND Y. WONG
}

(Communicated by Doug W. Curtis)

\begin{abstract}
Let $M$ be a compact connected Hilbert cube manifold ( $Q$-manifold). Define $C_{z}(M)$ to be the smallest integer $k$ such that $M$ can be covered with $k$ open subsets each of which is homeomorphic to $Q \times[0,1)$. Recently L. Montejano proved that, for every compact connected polyhedron $P, C_{z}(P \times$ $Q)=\operatorname{cat}(P)+1$, where $\operatorname{cat}(P)$ is the Lusternik-Schnirelmann category of $P$. Using a different approach, we prove a noncompact analog of the above theorem by showing that $C_{z}(P \times Q \times[0,1))=\operatorname{cat}(P)$ for every compact connected polyhedron $P$.
\end{abstract}

1. Introduction. For a compact polyhedron $P$, the Lusternik-Schnirelmann (L-S) category of $P$, cat $(P)$, is the smallest integer $k$ such that $P$ can be covered with $k$ subpolyhedra each of which is null homotopic in $P$. A $Q$-manifold is a separable metric space modeled on the Hilbert cube $Q=[0,1]^{\infty}$. Let $M$ be a connected $Q$-manifold. Define $C_{z}(M)$ to be the smallest integer $k$ such that $M$ can be covered with $k$ open subsets each of which is homeomorphic to $Q \times[0,1)$. Recently, L. Montejano proved the following theorem concerning the relationship between cat $(P)$ and $C_{z}(P \times Q)(P \times Q$ being a $Q$-manifold [WE]):

THEOREM $\left[\mathbf{M O}_{1}\right]$. For every compact connected polyhedron $P$,

$$
C_{z}(P \times Q)=\operatorname{cat}(P)+1 \text {. }
$$

Obviously, $C_{z}(Q)=2$. On the other hand, suppose that $M$ is a compact connected $Q$-manifold with $C_{z}(M)=2$. By the triangulation theorem of $\left[\mathbf{C H}_{1}\right], M$ is homeomorphic to $P \times Q$ for some compact connected polyhedron $P$. Hence $\operatorname{cat}(P)=1$ by the above theorem, i.e., $P$ is contractible. Thus $M$ is homeomorphic to $Q$, since the Hilbert cube is the only contractible compact $Q$-manifold $\left[\mathbf{C H}_{1}\right]$.

Extending to the noncompact case, we observe that if $P=\{$ point $\}$,

$$
C_{z}(P \times Q \times[0,1))=1=\operatorname{cat}(P) .
$$

Hence it is natural to ask if $C_{z}(P \times Q \times[0,1))=\operatorname{cat}(P)$ in general. In this note we answer the yuestion affirmatively.

THEOREM 1. For every compact polyhedron $P$,

$$
C_{\iota}\left(P \times Q \times{ }_{\iota}(0,1)\right)=\operatorname{cat}(P) .
$$

For an arbitrary space $X$, let $\operatorname{cat}^{\prime}(X)$ denote the smallest integer $k$ such that $X$ can be covered by $K$ open sets each of which is null-homotopic in $X$. For a

Received by the editors June 6, 1986 and, in revised form, November 12, 1986.

1980 Mathematics Subject Classification (1985 Revision). Primary 58E05, 57N20. Z-sets.

Key words and phrases. Lusternik-Schnirelmann category, Hilbert cube manifold, polyhedron, 
compact polyhedron $P$, it is easily seen that $\operatorname{cat}(P)=\operatorname{cat}^{\prime}(P)=\operatorname{cat}^{\prime}(P \times Q)$. This observation, together with the triangulation theorem refered to above, shows that Montejano's result and our result may be formulated as follows:

COROLLARY. For every compact connected $Q$-manifold $M, C_{z}(M)=\operatorname{cat}^{\prime}(M)$ +1 and $C_{z}(M \times[0,1))=\operatorname{cat}^{\prime}(M)$.

ProOF OF THEOREM 1 . It is easy to verify that $\operatorname{cat}(P) \leq C_{z}(P \times Q \times[0,1))$. On the other hand, suppose that cat $(P)=k$. Let $P_{1}, P_{2}, \ldots, P_{k}$ be a null-homotopic cover of $P$. Denote $M=P \times Q \times\{0\} \subset P \times Q \times[0,1)=\tilde{M}$. Let $B_{i}=P_{i} \times Q \times\{0\}$. $B_{i}$ is a null-homotopic Z-set of $\tilde{M}$. By standard I-D topology we may assume that the cone of $B_{i}$ is imbedded in $\tilde{M}$ as a $\mathbf{Z}$-set in such a way that the base coincides with $B_{i}$. Since the cone contracts to its vertex $v_{i}$ and $v_{i}$ has an open neighborhood $U_{i}$ homeomorphic to $Q \times[0,1)$, by Z-set unknotting there is a homeomorphism on $\tilde{M}$ taking $B_{i}$ into $U_{i}$. So, without loss of generality we may assume that $B_{i} \subset U_{i}$. Let $\left\{Q_{n} \mid n=1,2, \ldots\right\}$ denote the countable collection of all end-faces of $Q$; that is, each $Q_{n}=\pi_{i}^{-1}(0)$ or $\pi_{i}^{-1}(1)$ for some $i$, where $\pi_{i}$ is the $i$ th-projection of $Q$ into the $i$ th-factor. Let $A_{n m}=P_{i} \times Q_{n} \times[0, m /(m+1)]$, where $n, m=1,2, \ldots$, and let $A_{i}=P_{i} \times Q \times[0,1)$.

Each $A_{n m}$ is a Z $\mathbf{Z}$-set in $\tilde{M}$ and is contractible to $P_{i} \times Q_{n} \times\{0\} \subset U_{i}$. We want to employ the engulfing apparatus of $\left[\mathbf{C H}_{1}\right]$ to construct an open imbedding $h_{i}$ of $Q \times[0,1)$ into $\tilde{M}$ such that $\bigcup_{n, m} A_{n m} \subset h_{i}(Q \times[0,1))$. Let $g_{i}: Q \times[0,1) \rightarrow U_{i}$ be a homeomorphism. The key is to verify the following:

LEMMA A. For any Z-set $K \subset P_{i} \times Q \times[0, r], r<1$, there is an open imbedding $g_{i}^{\prime}: Q \times[0,1) \rightarrow \tilde{M}$ such that

$$
g_{i}^{\prime}\left|\left(Q \times\left[0, \frac{1}{2}\right]\right)=g_{i}\right|\left(Q \times\left[0, \frac{1}{2}\right]\right) \quad \text { and } \quad g_{i}^{\prime}\left(Q \times\left[0, \frac{2}{3}\right)\right) \supset K .
$$

ProOF OF LEMMA A. Let $K^{\prime}=K-g_{i}\left(Q \times\left[0, \frac{1}{2}\right)\right)$, and assume $K^{\prime} \neq \varnothing$. Since $P_{i} \times Q \times\{0\} \subset U_{i}, K^{\prime}$ is contractible to a subset of $g_{i}\left(Q \times\left[0, \frac{1}{2}\right)\right)$. Using the fact that $Q \times[0,1)$ is contractible to $Q \times\left[\frac{1}{2}, 1\right)$, we may assume that $K^{\prime}$ is homotopic to a subset of $g_{i}\left(Q \times\left[\frac{1}{2}, \frac{2}{3}\right]\right)$ and the homotopy lies in the $Q$-manifold $N=$ $\tilde{M}-g_{i}\left(Q \times\left[0, \frac{1}{2}\right)\right)$. Since $g_{i}\left(Q \times\left\{\frac{1}{2}\right\}\right)$ is a Hilbert cube and a Z-set in the Hilbert cube $g_{i}\left(Q \times\left[\frac{1}{2}, \frac{2}{3}\right]\right) \subset N$, using Z $\mathbf{Z}$-set unknotting we may replace the homotopy by another homotopy $\left\{\phi_{t}\right\}$ of $K^{\prime}$ into $N$ so that $\phi_{0}=$ identity, $\phi_{t} \mid K^{\prime} \cap g_{i}\left(Q \times\left\{\frac{1}{2}\right\}\right)=$ identity for all $t, \phi_{1}\left(K^{\prime}\right) \subset g_{i}\left(Q \times\left[\frac{1}{2}, \frac{2}{3}\right)\right)$ and for any $x \in K^{\prime}-g_{i}\left(Q \times\left\{\frac{1}{2}\right\}\right),\left\{\phi_{t}(x)\right\}_{t} \subset$ $N-g_{i}\left(Q \times\left\{\frac{1}{2}\right\}\right)$. By Z-set unknotting there is a homeomorphism $f: N \rightarrow N$ such that $f \mid g_{i}\left(Q \times\left\{\frac{1}{2}\right\}\right)=$ identity and $f\left(K^{\prime}\right) \subset g_{i}\left(Q \times\left[\frac{1}{2}, \frac{2}{3}\right)\right)$. Define $g_{i}^{\prime}$ by $g_{i}^{\prime}\left|\left(Q \times\left[0, \frac{1}{2}\right]\right)=g_{i}\right|\left(Q \times\left[0, \frac{1}{2}\right]\right), g_{i}^{\prime} \mid\left(Q \times\left[\frac{1}{2}, 1\right)\right)=f^{-1} g_{i} . g_{i}^{\prime}$ is what we wanted.

By Lemma $\mathrm{A}$ and the engulfing lemma of $\left[\mathbf{C H}_{1}\right]$ we can construct an open imbedding $\left.h_{i}: Q \times[0,1)\right) \rightarrow \tilde{M}$ such that $\bigcup_{n, m} A_{n m} \subset h_{i}(Q \times[0,1))$. Let $V_{i}=$ $h_{i}(Q \times[0,1))$ and $V=\bigcup_{i=1}^{k} V_{i}$. Then $V$ is an open set in $\tilde{M}$ containing the cap-set $P \times \bigcup_{n=1}^{\infty} Q_{n} \times[0,1)$; thus the complement $\tilde{M}-V$ is a Z-set $\left[\mathbf{C H}_{2}\right]$. It follows that $V$ and $\tilde{M}$ are homotopically equivalent, and therefore the manifold $V \times[0,1)$ and $\tilde{M}$ are homeomorphic. Since each $V_{i} \times[0,1)$ is homeomorphic to $Q \times[0,1), C_{z}(\tilde{M})=C_{z}(V \times[0,1)) \leq k$.

The theory extends to the following noncompact case. 
THEOREM 2. For every connected $Q$-manifold $M$,

$$
C_{z}(M \times[0,1))=\operatorname{cat}^{\prime}(M) .
$$

Proof. Since $\operatorname{cat}^{\prime}(M)$ is a homotopy invariant, $\operatorname{cat}^{\prime}(M)=\operatorname{cat}^{\prime}(M \times[0,1)) \leq$ $C_{z}(M \times[0,1))$. On the other hand, suppose $\operatorname{cat}^{\prime}(M)=k$. It can be easily shown that $M$ has a null-homotopic closed cover $C_{1}, C_{2}, \ldots, C_{k}$. Consider $M=M \times\{0\} \subset$ $M \times[0,1)$. As in the proof of Theorem A, each $C_{i} \subset U_{i}$ where $U_{i}$ is an open set homeomorphic to $Q \times[0,1)$. Let $g_{i}: Q \times[0,1) \rightarrow U_{i}$ be a homeomorphism. Denote $A_{n m}=C_{i} \times Q_{n} \times[0, m /(m+1)]$. By the same argument as in the proof of Lemma $\mathrm{A}$, there is an open imbedding $g_{i}^{\prime}: Q \times[0,1) \rightarrow \tilde{M}$ such that

$$
g_{i}^{\prime}\left|\left(Q \times\left[0, \frac{1}{2}\right]\right)=g_{i}\right|\left(Q \times\left[0, \frac{1}{2}\right]\right) \text { and } g_{i}^{\prime}\left(Q \times\left[0, \frac{2}{3}\right)\right) \supset A_{n m} .
$$

Following the same argument as that of Theorem 1 , we conclude that $M \times[0,1)$ is covered by $k$ open sets each of which is homeomorphic to $Q \times[0,1)$. Hence $C_{\boldsymbol{z}}(M \times[0,1)) \leq k$.

\section{REFERENCES}

[CH$\left.{ }_{1}\right]$ T. A. Chapman, Lectures on Hilbert cube manifolds, CBMS Regional Conf. Ser. in Math., no. 28, Amer. Math. Soc., Providence, R. I., 1975.

$\left[\mathbf{C H}_{2}\right]$ - Dense sigma-compact subsets of infinite-dimensional manifolds, Trans. Amer. Math. Soc. 154 (1971), 399-426.

[MO $\mathbf{M O}_{1}$ L. Montejano, Lusternik-Schnirelmann category and Hilbert cube manifolds, Preprint.

$\left[\mathbf{M O}_{2}\right]-$, A quick proof of Singhof's $\operatorname{cat}\left(M \times S^{1}\right)=\operatorname{cat}(M)+1$ theorem, Manuscripta Math. 42 (1983), 49-52.

[JA] I. M. James, On category, in the sense of Lusternik-Schnirelmann, Topology 19 (1978), 331-349.

[WE] J. E. West, Infinite products which are Hilbert cubes, Trans. Amer. Math. Soc. 150 (1970), 1-25.

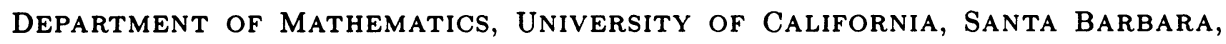
CALIFORNIA 93106 